\title{
Calcified Extradural Hematoma with overlying Subcutaneous Swelling: A Rare Case Report
}

\author{
Dr.Saloni Mehra, Dr.Anuj Kumar Tripathi, Dr. Abhishek Singh³, \\ Dr. Sameer R Verma 4
}

\begin{abstract}
Chronic ossified or calcified extradural hematomas are rare clinical entities presenting frequently in children.We report a rare case of Posttraumatic Chronic ossified extradural hematomas in a 35 years old male presenting two months after head injury with left occipito-suboccipital region swelling.

Keywords: calcification, extradural hematomas,head injury
\end{abstract}

\section{Introduction}

Calcified extradural hematomas are rare clinical entities presenting frequently in children. Although precise mechanism of an osseous transformation is still not well understood. Proposed hypothesis suggest that regressive and active processes are both major causes of ossification/calcification in these cases $(2,3)$. We report a rare case of Posttraumatic Chronic ossified extradural hematomas in a 35 years old male presenting two months after head injury with left occipitosuboccipital region swelling.

\section{Case report}

A 35 years old male presented in neurosurgery out door with left occipito-suboccipatal swelling with headache.On examination GCSscore was 15/15.Vitals were normal.Sensory and motor examination was normal .on local examination firm,non compressible ,nontender,nonpulsating swelling over left occipito-suboccipital region was present .He developed this swelling after fall from height two months back.After fall he developed transient loss of conciousness.There was no history of seizure,vomiting,ear nose or throat bleed.Noncontrast computed tomography scan revealed calcified extradural hematoma in left occipito-suboccipital region with hydrocephalus and left occipito-suboccipatal swelling. J shaped incision was given and excised the swelling which was firm and gritty in nature. Left occipitosuboccipitalcraniectomy was done and removed the calcified extradural collection. Post operative course was uneventful.

\section{Discussion}

Posttraumatic Chronic ossified extradural hematomas are rare entities.Ossified EDH is frequently present in paediatric age group (1). On review of literature, calcified chronic extradural hematoma has been reported in isolated case reports (1-3).

Although precise mechanism of an osseous transformation is still not well understood.Although precise mechanism of an osseous transformation is still not well understood, it has been hypothesized that damage to vascularized tissues such as bone and dura provokes inflammation, repair and remodeling in tissues (4). This natural sequence of healing is more rapid in children than in adults. Nakamura et al (5) and other investigators have proposed that chronic EDH starts with formation of fibroblast layer adjacent to durawithin four days after bleeding. This subsequently develops into connective tissue layer from hematoma margin to cranial vault, which undergoes hyalinization and subsequent calcium deposition under the condition of poor circulation or malabsorption of hematoma content (5). These may cause intra and extra cranial calcification.These is seen in our case in which calcified EDH with extra cranial firm occipital- suboccipital swelling was present.

\section{References}

1. Iwakuma T, Brunngraber CV. Extradural ossification followingan extradural hematoma.J Neurosurg 1974;441:104-6. Erdogan B, Sen O, Bal N, Cekinmez M, Altinors N. Rapidlycalcifying and ossifying epidural hematoma. PediatrNeurosurg 2003;39:208-11.

Mathuriya SN, Kak VK, Banerjee AK. Ossified epidural

hematomas: Report of two cases.ClinNeurolNeurosurg 1989; 91:269-72.

Buckwalter JA, Cruess RL. Healing of the masculoskeletal tissues; In Rockwood CA, Green DP, Bucholz RW (eds): Fractures in adults, ed 3, Philadelphia, Lippincott 1991; vol 1, pp 181-222

7. Nakamura N. The relationship between head injuries and chronic subdural hematoma. Brain Nerve 1966; 18:702-9. 


\section{Authors}

Dr. SaloniMehra - Resident general surgery, subharti medical college and university, meerut.

DrAnujkumartripathi - Assistant professor in neurosurgery division of general surgery, Subharti medical college and university, meerut.

Dr. Abhishek Singh - Resident general surgery, subharti medical college and university,meerut.

Dr. Sameer R verma professor in radiodiagnosisdepartment, subharti medical college and university, meerut.

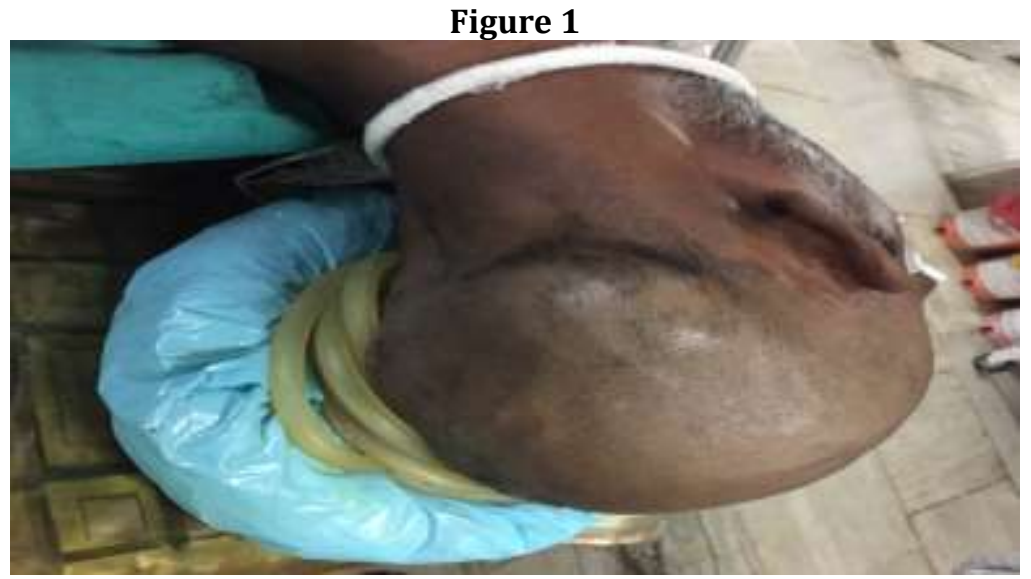

Figure showing left occipito-suboccipital swelling

Figure 2

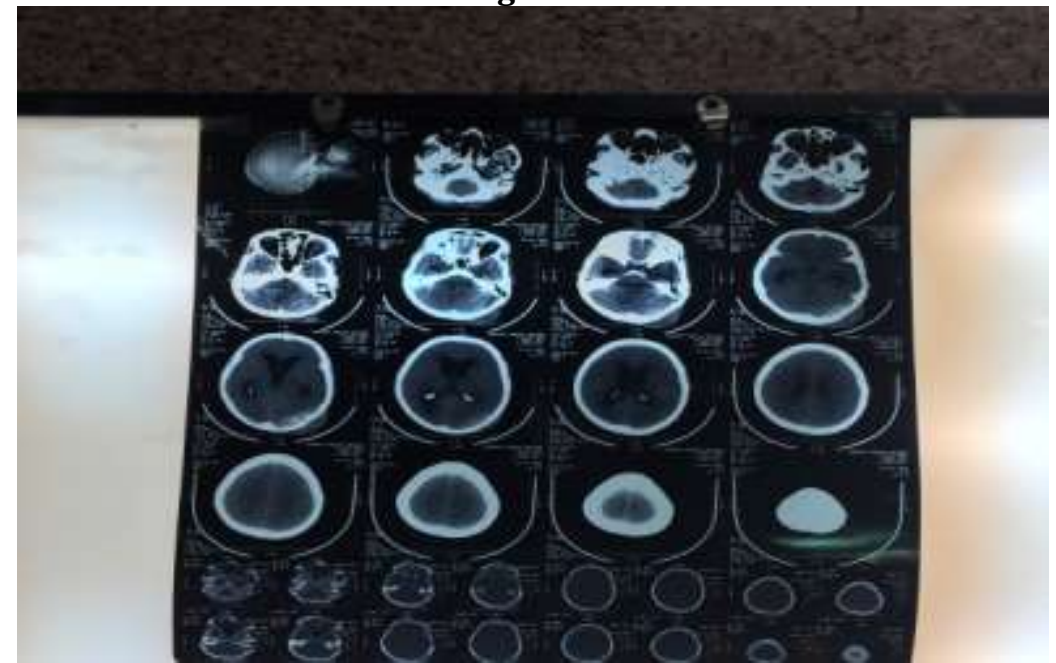

Computed tomography showing left occipito-suboccipital extra cranial swelling with extra dural hematoma

Figure 3 E

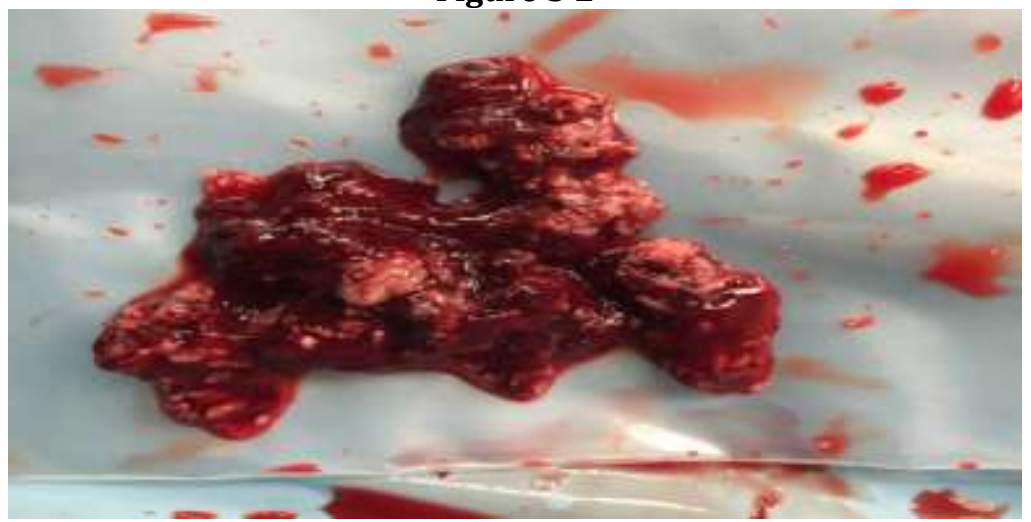

Figure showing calcified extra dural collection 
Figure 4

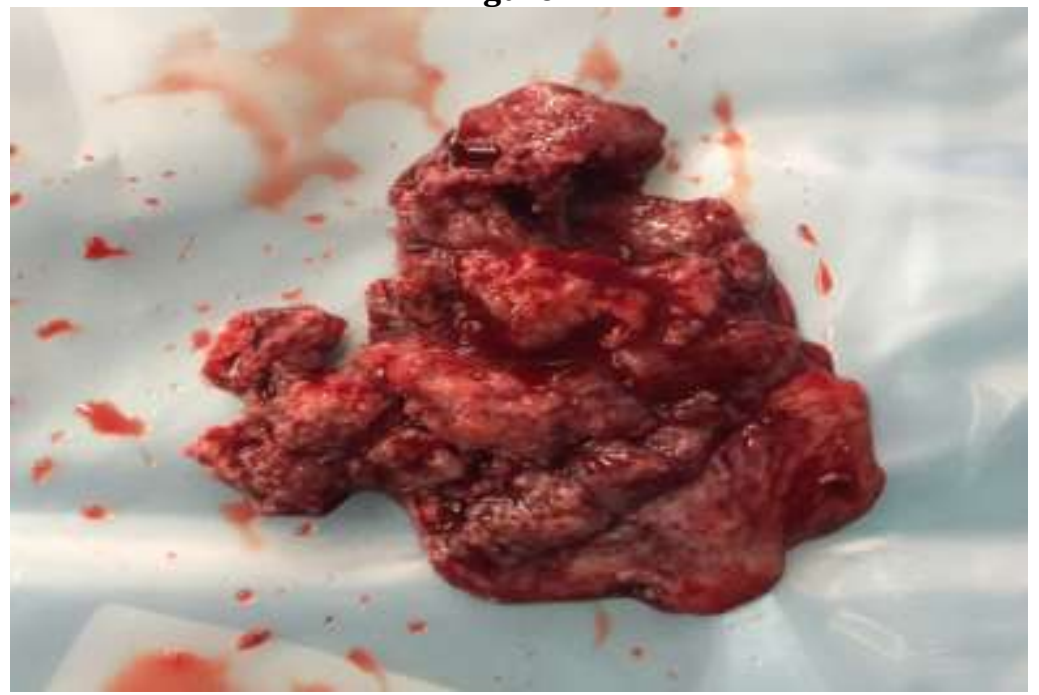

Figure showing extra cranial firm gritty collection 